\title{
Pensando y actuando en red. Análisis del hashtag \#QuédateEnCasa en México
}

\section{Thinking and acting in a network. Analysis of the hashtag \# QuédateEnCasa in Mexico}

Raul Anthony Olmedo Neri*

Universidad Nacional Autónoma de México

Facultad de Ciencias Políticas y Sociales

Circuito Mario de la Cueva s/n, Ciudad Universitaria,

C.P. 04510, Ciudad de México, México

Editor: Rogelio del Prado Flores

Fecha de recepción: 18 de mayo de 2020

Fecha de aceptación: 21 de julio de 2020 raulanthonyn@yahoo.com.mx

https://orcid.org/0000-0001-5318-0170

\section{RESUMEN}

El contexto generado por la pandemia de la CoviD-19 ha implicado que los gobiernos de cada Estado-Nación elaboren estrategias de comunicación en el espacio virtual para mantener a la población informada sobre el estado sanitario de cada país, así como de las medidas respectivas para su contención. En este sentido, el hashtag \#QuédateEnCasa forma parte de la estrategia comunicativa del gobierno mexicano para diseminar información oficial en la sociedad sobre el impacto de este reto epidemiológico.

Por ello, el presente trabajo aborda esta forma institucional de comunicación bajo el Análisis de Redes Sociales (ARS) con la finalidad de identificar la red que subyace en este hashtag, así como aquellos perfiles (nodos) que dentro

\footnotetext{
* Ingeniero Agrónomo especialista en Sociología Rural por la Universidad Autónoma Chapingo (UACh), licenciado en Ciencias de la Comunicación por la Universidad Nacional Autónoma de México (unam), maestro en Comunicación (UNAM). Con más de 30 artículos publicados en revistas nacionales e internacionales sobre las siguientes líneas de investigación: Economía Política de la Comunicación, Ecología Política; Comunicación Emergente; Megaproyectos. Ganador del tercer lugar en el Premio Internacional Gabriel Betancourt Mejía (Bogotá, 2014). Actualmente, consejero editorial de la revista Iberoamérica Social, donde publica mensualmente en el blog titulado Desde la Trinchera Sociológica.
} 
de ese entramado de interacciones se han posicionado dentro del flujo de información a partir de la cantidad de vínculos que poseen, de su capacidad de diseminar la información, así como el nivel de cohesión que se ha generado en dicha red.

Palabras clave: hashtag, twitter, \#quédateencasa, redes sociodigitales, ARS.

\section{ABSTRACT}

The context generated by the COVID-19 pandemic has implied that the governments of each Nation-State develop communication strategies in the virtual space to keep the population informed about the health status of each country as well as the respective measures for their containment. In this sense, the hashtag \#QuédateEnCasa is part of the Mexican government's communication strategy to disseminate official information in society about the impact of this epidemiological challenge.

For this reason, the present work addresses this institutional form of communication under the Social Network Analysis (ARS) in order to identify the network that underlies this hashtag, as well as those profiles (nodes) that within that network of interactions have been positioned within the information flow based on the number of links they possess, their ability to disseminate information, as well as the level of cohesion that has been generated in said network.

Keywords: hashtag, Twitter, \#quédateencasa, social media, SNA.

\section{INTRODUCCIÓN}

La propagación de la CoviD-19 a escala global tuvo consecuencias generales en el ámbito económico, político y social, aunque su impacto fue diverso en cada uno de los países afectados. Su capacidad de propagación en el ambiente implicó un cambio sustancial en el ámbito de la producción y reproducción de la vida social a nivel mundial, lo que obligó a cambiar el estilo de vida, destacando que "el efecto del virus no es tanto el número de personas que debilita o el pequeñísimo número de personas que mata. El efecto del virus es la parálisis relacional que propaga" (Berardi, 2020, p. 38). 
El impacto dentro del espacio geográfico derivó en llevar gran parte de las actividades cotidianas al mundo virtual; si bien ya existía un proceso convergente, la necesidad del distanciamiento social sirvió como elemento para acelerar las relaciones laborales vía internet; el home office o teletrabajo, así como las clases virtuales fueron elementos que incrementaron su presencia en la vida cotidiana. La necesidad sanitaria del distanciamiento espacial dentro de los procesos de socialización y el confinamiento de gran parte de la población también resultó en un incremento del uso de las redes sociodigitales, particularmente en el caso mexicano ese incremento fue de $42 \%$ y específicamente en Facebook, YouTube, WhatsApp y Twitter, entre otras (Gutiérrez, 2020), por lo que este conjunto de plataformas se convirtieron en un canal donde no solo se incrementó la cantidad de interacciones, sino el flujo de información apócrifa y fake news que han generado marcos de referencia equivocados y percepciones erróneas sobre el impacto de la Covid-19 en el país.

En este sentido, la potencialidad de las redes sociodigitales como canales de largo alcance e impacto directo en la población derivó en la creación de una estrategia comunicativa convergente emanada del gobierno federal mexicano que aprovechó la arquitectura reticular de internet para incrementar el número de población informada sobre las acciones y hacer frente al flujo de información falsa que venía reproduciendo un cuestionamiento sobre las medidas e incluso, en casos extremos, poniendo en duda la existencia del propio covid-19 (Luna, 2020).

De esta manera \#QuédateEnCasa se integra junto a \#SusanaDistancia, \#Mexicocovid19 y las conferencias impartidas por el Dr. Hugo López-Gatell como la estrategia empleada por el gobierno federal mexicano para atender en términos comunicativos las demandas informacionales de la población mexicana respecto a la pandemia. Por ello, el presente trabajo aborda el caso particular del hashtag \#QuédateEnCasa a partir del método de Análisis de Redes Sociales (ARS) con la finalidad de visualizar el alcance en términos de nodos (perfiles/cuentas) y vínculos (interacciones en Twitter a partir de la información) que se desarrollaron alrededor de este hashtag.

En este sentido, se ha empleado el software NodeXL para construir una base de datos sobre las interacciones en torno a dicho hashtag y calcular indicadores internos y externos de la red como el nivel de grado, la densidad de red y el grado de intermediación que permiten identificar aquellos nodos que poseen una posición estratégica dentro del flujo de la información alrededor de \#QuédateEnCasa. 
Para ello, el artículo se ha construido de la siguiente manera: primero se expone el marco teórico-analítico entre redes sociodigitales y flujo de información; en segundo lugar se plantea la metodología empleada en el ARS que comprende periodo de análisis, definición de indicadores y proceso de construcción de la base de datos; después se presentan los resultados obtenidos para su análisis; le sigue una pequeña discusión sobre los hallazgos encontrados y la construcción simbólica a la par del hashtag; y finalmente, se da paso a las conclusiones derivadas del presente estudio.

\section{MARCO TEÓRICO}

La relevancia e impacto de redes sociodigitales en la vida cotidiana deriva de su incorporación en las prácticas culturales, políticas, educativas, económicas y sociales que el individuo realiza en sociedad. La estructura descentralizada, multimodal, oblicua y reticular que posee internet y las redes sociodigitales (Facebook, Twitter, YouTube, Instagram, WhatsApp, entre otras), sumado al discurso de la libertad y la construcción de una cultura de la conectividad (Van Dijck, 2016) que se les dota, hace que estos sitios se vuelvan espacios digitales fascinantes, funcionales y amplificadores de los sentidos individuales y las percepciones colectivas.

Esto, no obstante, ha desencadenado una suerte de presión social de ser/ estar en el espacio digital debido a su bajo costo económico, su facilidad de uso y sobre todo la posibilidad de ampliar contactos, crear/compartir información, así como el incremento del marco de referencia de ser/estar en sociedad. Por tanto, "las redes digitales en particular y la Internet en general, se han convertido en el nuevo opio del pueblo ya que su fascinación es la fuerza que ata a los sujetos y los condiciona a ingresar en ese nuevo mundo" (García \& Olmedo, 2019, p. 90).

De hecho, con el paso del tiempo estas redes sociodigitales se han convertido en medios/contextos donde los individuos además de contribuir con la información que allí circula, establecen procesos de socialización mediante el aprovechamiento de las lógicas operativas de cada una de esas plataformas; ser amigo de alguien, seguir/ser seguido, dar un 'me gusta', bloquear o eliminar personas se convierten en parte de las novedosas formas de socialización en dichos espacios, donde la posibilidad de ser/estar pasa a ser una necesidad forzada por la constante innovación tecnológica y la presión social de 'encontrarse en la red'. Dada la fascinación social de estos espacios virtuales, la presentación y representación en 
estas plataformas derivan en un gran número de personas que pueden ser contactadas, informadas o atendidas de manera más rápida, eficaz y eficiente en tanto se trascienden las limitantes del espacio geográfico y se eliminan los intermediarios informativos (Han, 2016) que representan los medios de comunicación masiva tradicionales como la prensa, radio y televisión.

Estos conglomerados mediáticos lejos de ostentar una postura renuente ante este espacio-no-físico (García \& Olmedo, 2019) se han enfocado en realizar un proceso de convergencia en dicho espacio para no perder su carácter masivo e incluso incrementarlo. De allí que periódicos, estaciones de radio y de televisión han comenzado con procesos para estar presentes en este infinito campo digital.

Lo anterior adquiere relevancia dado el contexto generado por la restricción espaciotemporal de la CoviD-19; no obstante, la limitación espacial no eliminó el distanciamiento social, sino que redujo los procesos de socialización en el espacio público y los replicó en el mundo virtual. De esta manera internet y las redes sociodigitales se han convertido en sitios donde la gente no solo se informa, sino también interactúa y realiza parte de sus actividades cotidianas. Este proceso de adaptación fue previsto por el Estado mexicano antes de la pandemia, por lo que la construcción de canales dentro de estos espacios digitales se aceleró con la finalidad de aprovechar sus características y así incrementar el alcance de la información oficial en la población sobre el brote epidemiológico.

La acción anterior profundizó la necesidad de evidenciar la participación del Estado mexicano en internet, ya no solo como parte de su estrategia de e-gobierno (Criado \& Gil-García, 2013), sino para poder informar e interactuar de manera 'directa' con la población sobre la coviD-19 en estas plataformas sociodigitales. Cabe destacar que la presencia del Estado mexicano en internet no es derivada de la contingencia sanitaria, por el contrario, el inicio de la participación del gobierno mexicano (a nivel federal y estatal) en internet tiene un origen conflictivo que se remonta a 1994, particularmente con el levantamiento del Ejército Zapatista de Liberación Nacional (EZLN), dado que ese movimiento social altermundista tuvo un impacto nacional e internacional derivado de la diseminación de sus objetivos en el espacio digital por parte de un simpatizante estadounidense a dicho movimiento (Rodríguez, 2015; Rovira, 2017).

Con este antecedente, el gobierno federal inició con el proceso para poseer una página web, el cual pudo concretarse hasta 1996 (Rovira, 2017), de allí, paulatinamente las instituciones y los gobiernos comenzaron con este proceso de digitalización de trámites, atención y presencia; con la llegada de las redes socio- 
digitales, los gobiernos y sus respectivas instituciones tuvieron que realizar un segundo proceso de convergencia enclavado en estas plataformas. El uso de las redes sociodigitales por parte del gobierno se funda en un proceso más interactivo e informativo que operativo ya que se puede "pulsar de manera inmediata cuál es la opinión de la ciudadanía sobre el desempeño gubernamental, su uso no es sinónimo de modernidad per se, sino es un primer paso hacia la apertura" (Contreras, 2017, p. 21).

Este proceso no significa que las estrategias offline de comunicación oficial hayan sido desplazadas, sino que han entrado en un proceso de convergencia: comunicados publicados y compartidos en redes sociodigitales, la colocación de videos en canales institucionales, así como la transmisión en streaming de conferencias/ruedas de prensa y reuniones, constituyen formas de diseminación de información que aprovechan las lógicas operativas de cada plataforma para materializar un objetivo, en este caso institucional alrededor de la información.

Particularmente, esta segunda ola de presentación y representación institucional en México ha sido más rápida en tanto que para 2017,31 de los 32 gobiernos estatales poseían al menos cuentas oficiales en Facebook y Twitter (Contreras, 2017), lo que muestra que estas plataformas no solo han seducido a grandes sectores poblacionales, sino que han orillado a los gobiernos a replicar su presencia y participar en su lógica de funcionamiento con la finalidad de incrementar el impacto de su estrategia de comunicación.

De hecho, la forma en que las redes sociodigitales se insertan dentro de la relación sociedad-Estado adquiere relevancia no solo por la capacidad de diseminación de información, sino su incorporación dentro de procesos paralelos derivados del sistema democrático y sus mecanismos. Por ende, "los medios de comunicación on line son una parte del gran mundo social, político y cultural, que se entrelaza con la vida cotidiana de los individuos y con el funcionamiento de grupos, organizaciones e instituciones" (Dah LGren, 2012, p. 52). Así, la vinculación ejercida en estos espacios abre la posibilidad de establecer nuevos procesos, información y participación pública que se insertan entre la vida pública y la política como es la rendición de cuentas, la inmediatez y alcance de las publicaciones que allí realizan políticos, partidos, instituciones y gobiernos, las estrategias gubernamentales para poder mitigar el impacto generado por riesgos epidemiológicos como la pandemia, así como desastres naturales y todo aquel suceso en que el Estado tenga la obligación de informar a la sociedad. 
El caso del hashtag \#QuédateEnCasa es muestra de la apropiación institucional de estas redes para poder llegar a una mayor cantidad de personas, por lo que su estudio debe visualizar el alcance y las estructuras que se forman alrededor del flujo de información generado. En este caso, estudiar esta estrategia desde el Análisis de Redes Sociales (ARS) implica pensar el impacto no solo en términos del número de personas que interactuaron con \#QuédateEnCasa, sino identificar la relevancia de ciertos perfiles/cuentas que lo promovieron y que lo diseminaron con la finalidad de evaluar y, en su caso, redireccionar las estrategias institucionales.

\section{METODOLOGíA}

El Análisis de Redes Sociales (ARS) es un método que ha sido utilizado en las ciencias sociales para analizar las estructuras que se generan en un grupo social determinado a partir de los vínculos que crean en sus procesos de socialización. De esta manera, este método "se centra en las relaciones entre los actores, y no en los actores individuales y sus atributos" (Hanneman, 2000, p. 7), por lo que carácter analítico se forma a partir de la interacción entre individuos mediante lazos que han construido a lo largo de su devenir social.

El uso de este método en el ámbito social ha estado vinculado a la construcción de redes a partir de variables emocionales, construcciones sociales, percepciones subjetivas, líneas de parentesco, estructuras de poder y flujos de información; por ende, este cuerpo metodológico está constituido por los nodos (usuarios/actores/perfiles) y los vínculos que crean entre sí (sean de amistad, de interacción, de comunicación, de parentesco, o poder, por ejemplo). Cuando se selecciona una variable dentro de un grupo, se genera entonces la red, es decir, el conjunto de lazos y estructuras que se desarrollan bajo una variable particular que delimita tanto la forma como la dinámica de dicha estructura reticular. De esta manera, los enlaces son el núcleo de las redes ya que ellos le dan forma y función de la red, por lo tanto "no son más que la representación de flujos, de intercambios, de interacciones y de los movimientos complejos que resultan de todos ellos" (Pisani \& Piotet, 2007, p. 57).

De manera particular, el ARS ha estado presente desde la noción de web 1.0 como método de análisis del espacio virtual (Rogers, 2018); los nodos son los perfiles de los usuarios, mientras que los vínculos pueden ser la lista de amigos 
que posee un perfil, la dicotomía de seguir/seguir seguido en una red o por las estructuras que se generan mediante la interacción de nodos con la información que fluye de manera permanente y a veces redundante.

Esta última forma es la que se ha empleado en este estudio en tanto el uso del hashtag \#QuédateEnCasa dentro de comentarios, tuits, retuits y réplicas en Twitter permite rastrear esas relaciones de interacción-comunicación, las cuales se definen como "aquellas relaciones sociales efímeras/perdurables que se desarrollan entre individuos mediante el flujo de información que en estas plataformas se lleva a cabo de manera oblicua, permanente y redundante" (Olmedo, 2020 a, p. 75).

Los estudios del Análisis de Redes Sociales aplicados en hashtag cada vez son más empleados; sea para el análisis de estrategias convergentes de movimientos sociales (Olmedo, 2019), iniciativas sociales en contra de alguna reforma en materia de telecomunicación e internet (Torres, 2013) o para evaluar campañas digitales comerciales o políticas, la hibridación del ARs con los hashtags adquiere un papel novedoso y necesario dado que cada hashtag es una construcción simbólica y semántica específica que responde a un contexto o demanda particular, en consecuencia la red que se forma alrededor de ellos es estructuralmente diferente y única. Son las relaciones de interacción-comunicación las que dan forma a la red y por las cuales algunos nodos se posicionan estratégicamente en dicha estructura reticular.

Para este análisis, se ha empleado el software NodeXL con la finalidad de extraer los nodos y vínculos alrededor de dicho hashtag desde el 23 de marzo y hasta el 26 de abril de 2020; aunque el hashtag también ha estado presente en Facebook, las modificaciones realizadas en 2014 removieron la posibilidad de extraer este tipo de información mediante el uso de Application Programming Interface (API) (Rogers, 2018), por lo que el análisis se ha enfocado en Twitter en tanto esta opción es viable siempre y cuando no se violenten los términos y condiciones que provee esta plataforma. Para visualizar la red se ha utilizado el programa Gephi, el cual permite realizar visualizaciones más estéticas ytambién calcular los indicadores (Ricaurte \& Ramos-Vidal, 2015).

Además de ello, la base de datos final se construyó a partir de la ubicación de dichos nodos, por lo que se delimitó la red a todas aquellas interacciones desarrolladas en Twitter entre nodos con ubicación en el territorio mexicano, en tanto se encontró que este hashtag también ha sido utilizado en diferentes países de América Latina y España, lo que demuestra la relevancia del idioma como vinculador más allá de las fronteras político-administrativas. De esta manera, la red 
adquiere un carácter territorial, que incentiva el análisis de este tipo de estudios interdisciplinarios entre la comunicación y el territorio.

La estructura y dinámica interna de la red pueden ser analizadas a partir de indicadores que han sido construidos derivado de la teoría de grafos (Lozares, 1996), una aportación emanada de las ciencias Matemáticas y Computacionales a este método. Los indicadores que serán utilizados en este trabajo se encuentran en la siguiente tabla.

TABLA 1. INDICADORES DE RED

\begin{tabular}{|l|l|}
\hline \multicolumn{1}{|c|}{ INDICADOR } & \multicolumn{1}{c|}{ DESCRIPCIÓN } \\
\hline Tipo de red & $\begin{array}{l}\text { Debido a la capacidad direccional de los vínculos la red puede ser dirigida. Si } \\
\text { solo se quiere expresar la mera existencia de vínculos, entonces la red será } \\
\text { no dirigida. }\end{array}$ \\
\hline Densidad de red & $\begin{array}{l}\text { "Es la relación entre el número de líneas existentes dividido por el número } \\
\text { de líneas posibles" (Paniagua, 2012, p. 37). }\end{array}$ \\
\hline Nivel de grado & $\begin{array}{l}\text { En grado refiere la cantidad de vínculos que posee un nodo dentro de la red. } \\
\text { Si los vínculos poseen direccionalidad, este indicador puede desagregarse } \\
\text { en grado de salida y de entrada. }\end{array}$ \\
\hline Grado de intermediación & $\begin{array}{l}\text { "La intermediación mide las veces que un nodo se interpone entre todo en } \\
\text { su distancia geodésica" (Paniagua, 2012, p. 57). }\end{array}$ \\
\hline Distancia geodésica & \begin{tabular}{l} 
Es el camino más corto para conectar dos nodos cualquieras en la red. \\
\hline
\end{tabular}
\end{tabular}

FUENTE: ELABORACIÓN PROPIA.

Finalmente, resulta necesario anotar la profundidad de análisis del presente estudio debido a que "hay dos formas de hablar de una red. La primera hace referencia a los nodos y a los enlaces que los relacionan, y la segunda insiste en los flujos que circulan" (Pisani \& Piotet, 2007, p. 58).

De esta manera, el análisis aborda ambas formas en tanto no solo basta conocer la estructura de la red, sino también el flujo de información que circula en dicha estructura reticular. Lo anterior permite contextualizar los indicadores alrededor del hashtag, así como identificar aquellos nodos que adquieren un pa- 
pel estratégico, esto es, que poseen una posición relevante dentro del flujo de información, sea porque ellos son los promotores del hashtag o porque posee una capacidad de diseminación de información mayor a la de otros nodos en la red.

Así pues, no basta con conocer la estructura de la red y sus indicadores, sino contextualizar los nodos y el flujo de información para entender cuál es la lógica de funcionamiento de dicha información en el grupo socialmente delimitado y geográficamente acotado.

\section{RESULTADOS Y ANÁLISIS}

A partir de la información obtenida, se ha observado que durante el periodo de recolección de información se identificó un total de 16982 nodos y 40422 vínculos que interactuaron con el hashtag, de los cuales 7998 nodos y 10773 vínculos se encuentran en el territorio mexicano. Esto significa que la participación e interacción de nodos con el hashtag \#QuédateEnCasa en el México representa el 47.1\% de nodos y el $26.65 \%$ de vínculos totales.

La red circunscrita al territorio mexicano es de tipo dirigida, es decir, que se conoce la direccionalidad de los enlaces creados entre los nodos; con este atributo es posible determinar el flujo de información en la red. El grado de cohesión en esta red se obtiene en términos porcentuales al calcular la densidad de red; en esta red dicha densidad es de $0.01 \%$, lo que significa que esta estructura no está cohesionada ${ }^{1}$.

La poca cohesión que existe en esta red encuentra razón en su función, esto es de diseminación de información, por lo que esta red no está hecha para consolidar vínculos permanentes entre los nodos dentro de Twitter, sino difundir la información al mayor número de personas. Por ende, este tipo de redes "propagan ideas; ellas propagan conocimiento; ellas propagan influencia” (Barabási, 2011, p. 12), por lo tanto, el objetivo de esta estrategia no es fortalecer los vínculos existentes entre la población y el gobierno federal, sino crear enlaces débiles (Granovetter, 1973) entre otros usuarios que no necesariamente están vinculados a las instituciones de manera constante, con la finalidad de hacer llegar a través de ese vínculo

1 Si bien los nodos pueden estar vinculados mediante la lógica de seguir/ser seguido dentro de Twitter, en esta red no se refleja esa variable en tanto los vínculos se generan a partir de la publicación, réplica o etiquetar a personas con este hashtag, por lo que puede ser que dos nodos sí posean un vínculo de "relación social", pero no se muestra en el flujo de información. 
débil la información a un número de personas que pueden o no estar al tanto de esta campaña o la información institucional al respecto.

En la siguiente imagen se muestra la red de interacción-comunicación que se ha generado en el territorio mexicano. En esta imagen se ha establecido el criterio de grado, por lo que aquellos nombres de cuentas que aparecen son las que poseen el mayor número de enlaces dentro de la red.

FIGURA 1. RED \#QUÉDATEENCASA A PARTIR DEL NIVEL DE GRADO

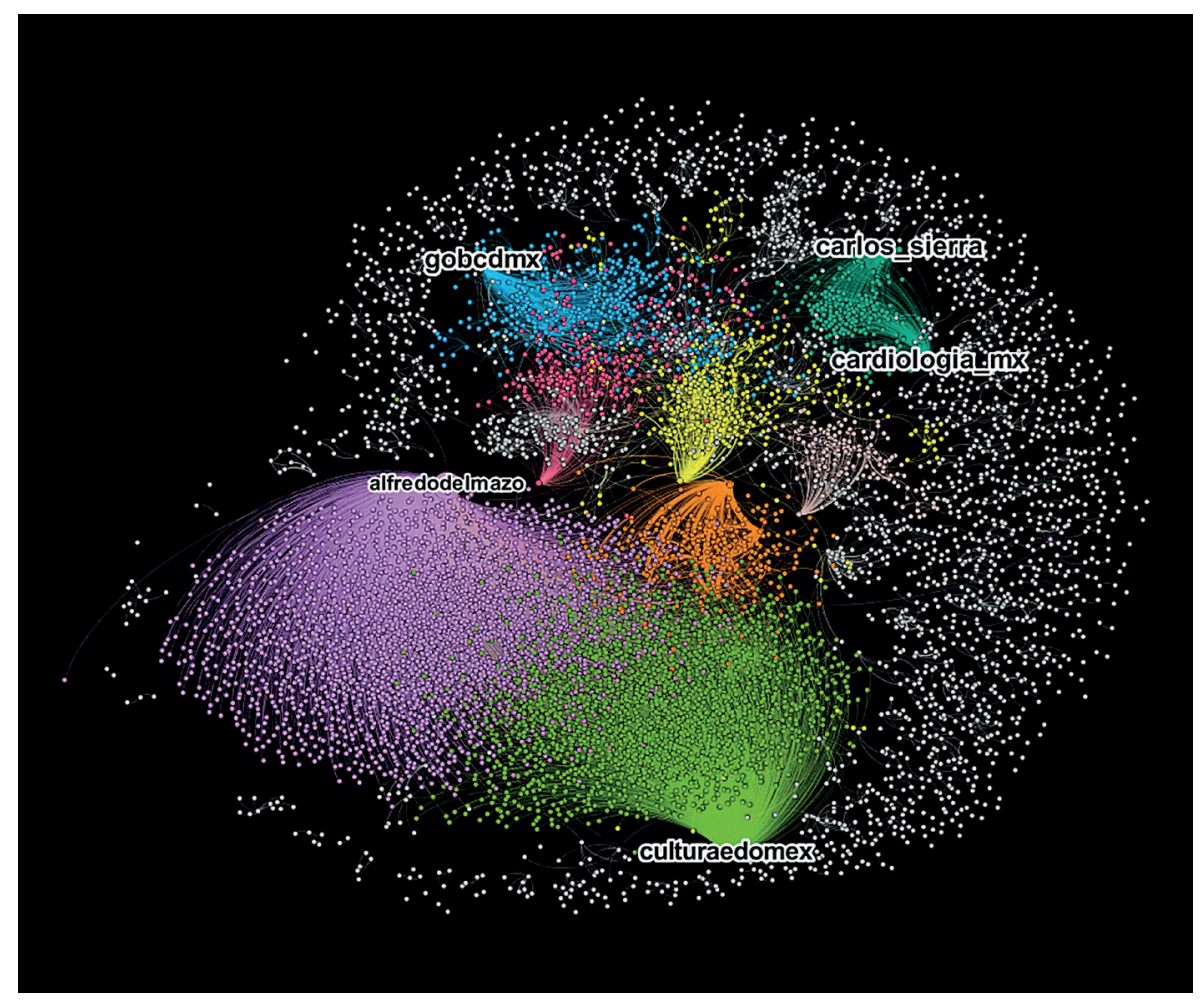

Fuente: ELABORACIÓN PROPIA CON DATOS DE NODEXL Y GEPHI.

La visualización se realizó bajo el algoritmo Force Atlas 2, mientras que el uso de colores se debe a la presentación generada por el indicador de modularidad, por el que se identifican las redes dentro de la red, es decir, los subgrafos que se generan internamente. Cada grupo está estructurado bajo la publicación de un nodo particular; así, los nodos que interactuaron con las publicaciones de @alfredodelmazo no lo hicieron con las publicaciones de @cardiologia_mx o @gobcmdx, por ejemplo. 
Cada subgrafo (grupo de nodos vinculados entre sí y sin o con muy poca vinculación con otros nodos de la red) es ciertamente autónomo; dicha autonomía descansa en que los usuarios no poseen vinculación alguna con los otros nodos, lo cual responde parcialmente a que las redes sociales conformadas por amigos, familia, compañeros de trabajo o páginas/individuos que sigue cada individuo no se vinculan con estas otras cuentas que también están generando información al respecto.

Por lo anterior, esta parcial limitación en cuanto a cuentas institucionales que dan información sobre estos temas es lo que posiciona estratégicamente a esos cinco nodos; la estructura de estos subgrafos es de tipo estrella, es decir, que existe un nodo fundamental del que emana la red/subgrafo, por lo que sin él, los nodos a su alrededor no podrían establecer una vinculación.

De esta manera, si se eliminara el nodo@alfredomazo, @gobcdmx,@cardiología_mx, @culturaedomex y @carlos_sierra, la red se visualizaría como un conjunto de puntos sin vinculación alguna y dispersos en la infinitud en la red. Entonces, la posición particular de estos nodos es que ellos son los que le dan forma a la red. En la siguiente tabla se muestran los cinco nodos con mayor nivel de grado dentro de la red.

TABLA 2. NODOS CON MAYOR NIVEL

DE GRADO EN LA RED \#QUÉDATEENCASA

\begin{tabular}{|l|l|c|c|c|c|}
\hline \multicolumn{1}{|c|}{ NODO } & \multicolumn{1}{|c|}{ ROL } & $\begin{array}{c}\text { GRADO DE } \\
\text { ENTRADA }\end{array}$ & $\begin{array}{c}\text { GRADO DE } \\
\text { SALIDA }\end{array}$ & $\begin{array}{c}\text { NIVEL DE } \\
\text { GRADO }\end{array}$ & $\begin{array}{c}\text { NIVEL DE } \\
\text { INTERM EDIACIÓN }\end{array}$ \\
\hline Alfredodelmazo & Político & 3121 & 1 & 3122 & 0 \\
\hline Culturaedomex & Institución pública & 1665 & 4 & 1669 & 4696 \\
\hline Gobcdmx & Institución pública & 181 & 3 & 184 & 557 \\
\hline Cardiología_mx & Institución médica & 170 & 0 & 170 & 0 \\
\hline Carlos_sierra & Médico & 169 & 1 & 170 & 0 \\
\hline
\end{tabular}

Fuente: ELABORACIÓN PROPIA CON DATOS DE GEPHI. 
Como se observa, de los cincos nodos más relevantes en términos del número de enlaces que posee destaca el gobernador del Estado de México, así como tres instituciones y un profesional médico. La lógica del funcionamiento entre los grados de entrada y salida resultan útiles para conocer la dinámica de interacción que se ha dado alrededor de un hashtag. Los tuits y comentarios que emanan del nodo son grados de entrada, mientras que los retuits y las etiquetas del nodo representan los grados de salida; así, mientras que el gobernador Alfredo del Mazo realizó un tuit junto al hashtag \#QuédateEnCasa, él fue retuiteado o etiquetado en publicaciones junto a dicho hashtag 3121 veces. Esta cantidad de interacciones no necesariamente tienen que ver con la publicación hecha, sino que pueden derivar de publicaciones donde etiquetan al nodo debido a su posición política, cultural o comunicación; en este caso al cargo político que ostenta y su relevancia en el espacio público del Estado de México.

Esta interacción y cantidad de vínculos poseen relevancia dado el rol que juega cada una de estas cuentas en el plano real concreto; tanto el político como las instituciones forman parte de la esfera pública, por lo que su función no solo se da por la pertinencia de su actuar, sino por el carácter informativo que adquieren durante la pandemia. El caso del médico (@carlos_sierra), él pertenece al Instituto Nacional de Cardiología, por lo que aunque su papel también es público, no tiene el mismo impacto que los otros nodos.

Ahora bien, este escenario tiene una razón que se explica mediante el contexto y el proceso de organización institucional sobre la pandemia, ya que, si bien el hashtag \#QuédateEnCasa no se articuló en el ámbito federal desde un inicio, con las medidas empleadas a lo largo del territorio esta expresión creativa se volvió parte del plan de comunicación del gobierno presidido por Andrés Manuel López Obrador (AMLO). De hecho, el primer tuit publicado durante el periodo analizado está fincado en una institución del Estado de México, el cual se muestra en la figura 2.

La fecha se vincula con el lanzamiento de la campaña realizada en la Ciudad de México (Cruz, 2020), así como las medidas tomadas para reducir la aglomeración social en espacios públicos y comerciales que se propusieron a nivel federal. Conforme la pandemia incrementó el número de contagios y defunciones, la necesidad de vincular las estrategias estatales con el gobierno federal derivó en la apropiación del hashtag dentro de las conferencias impartidas por el Dr. Hugo López-Gatell (Ramírez, 2020). 
FIGURA 2. TUIT CON HASHTAG \#QUÉDATEENCASA

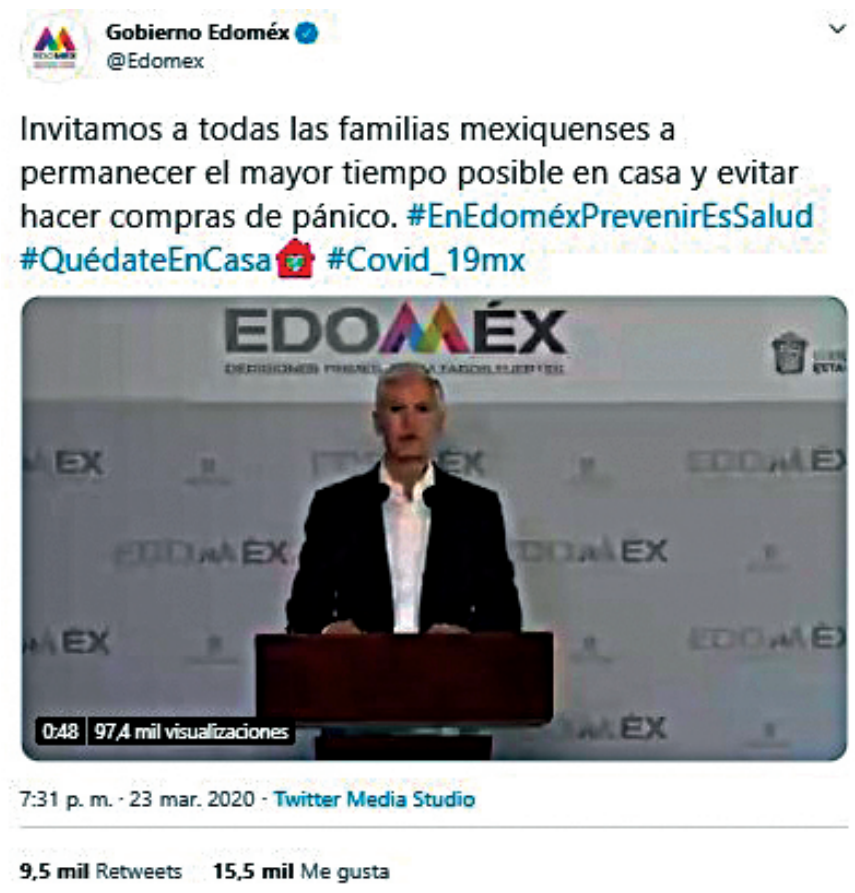

FuENTE: https://twitter.com/edomex/status/1242262789856677888

Dicha incorporación no solo se hizo en las conferencias sobre la coviD-19, sino también en su utilización por parte de diversas instituciones, líderes de opinión e incluso en la propia cuenta de López-Gatell; además, se creó un sitio web del gobierno federal en donde se da información pública y oficial sobre el impacto de esta pandemia y las medidas tomadas para mitigar el impacto. En las siguientes figuras se puede observar tanto el uso del hashtag como su implementación en la página oficial mencionada.

Aunque en estos nodos posean una alta cantidad de vínculos, lo cierto es que solo dos instituciones muestran un grado de intermediación (@culturaedomex y @gobcdmx), lo que permite observar que los indicadores poseen una autonomía entre sí. De esta manera, ostentar un alto nivel de vínculos no necesariamente significa que dicho nodo sea un diseminador o promotor de la información en la red. De allí que el grado de intermediación ofrezca una mirada sobre el flujo de información en dicha red, ubicando aquellos nodos que poseen una posición estratégica en dicho flujo. 
FIGURA 3. PÁGINA WEB SOBRE INFORMACIÓN DE LA COVID-19

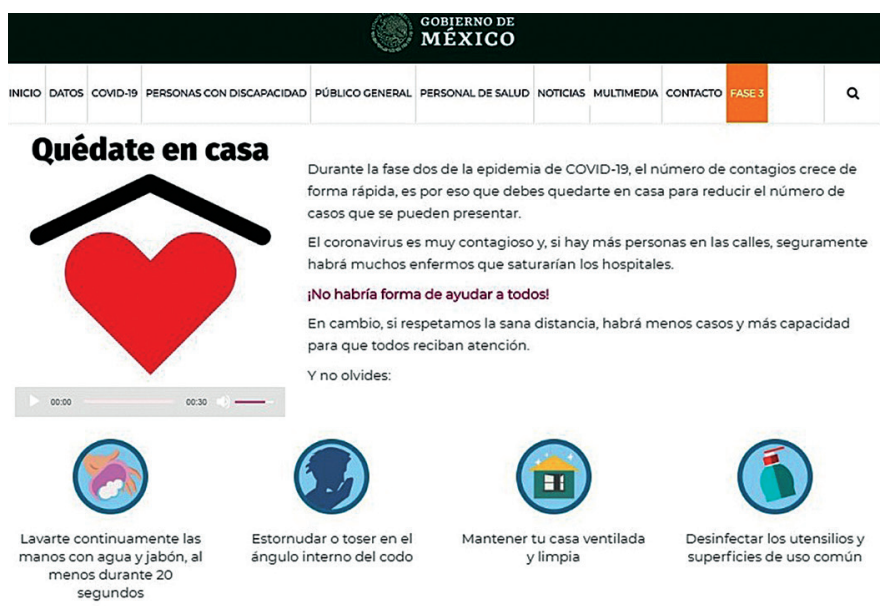

FUENTE: https://coronavirus.gob.mx/quedate-en-casa/

FIGURA 4. USO DEL HASHTAG \#OUÉDATEENCASA POR AUTORIDADES DEL GOBIERNO FEDERAL

Hugo López-Gatell Ramírez

@HLGatell

Reconocemos el esfuerzo del @Conacyt_MX para tener en mayo 700 ventiladores mecánicos económicos, de alta calidad y seguridad biomédica, fabricados con ciencia y tecnología nacionales. Lo fundamental es prevenir, evitar que personas graves necesiten estos equipos. \#QuédateEnCasa 중.

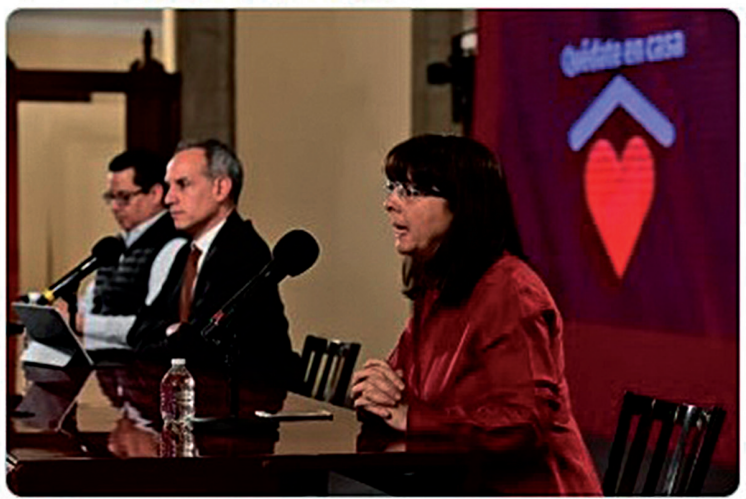

8:44 p. m. $\cdot 23$ abr. 2020 - Twitter Web App

1,8 mil Retweets 6.4 mil Me gusta

FUENTE: https://twitter.com/hlgatell/status/1253500061230739456 
Este indicador adquiere relevancia desde la perspectiva comunicativa ya que a partir de la direccionalidad de los nodos se pueden construir caminos para conectar a dos nodos cualesquiera en la red. Aquellos nodos que poseen un alto grado de intermediación tienen la capacidad de posicionarse estratégicamente en la red dado que una cantidad relevante del flujo de información necesita de ellos para llegar a un destino específico.

De esta manera, la siguiente figura muestra la red a partir del grado de intermediación.

FIGURA 5. RED DE INTERACCIÓN-COMUNICACIÓN A PARTIR DEL GRADO DE INTERMEDIACIÓN

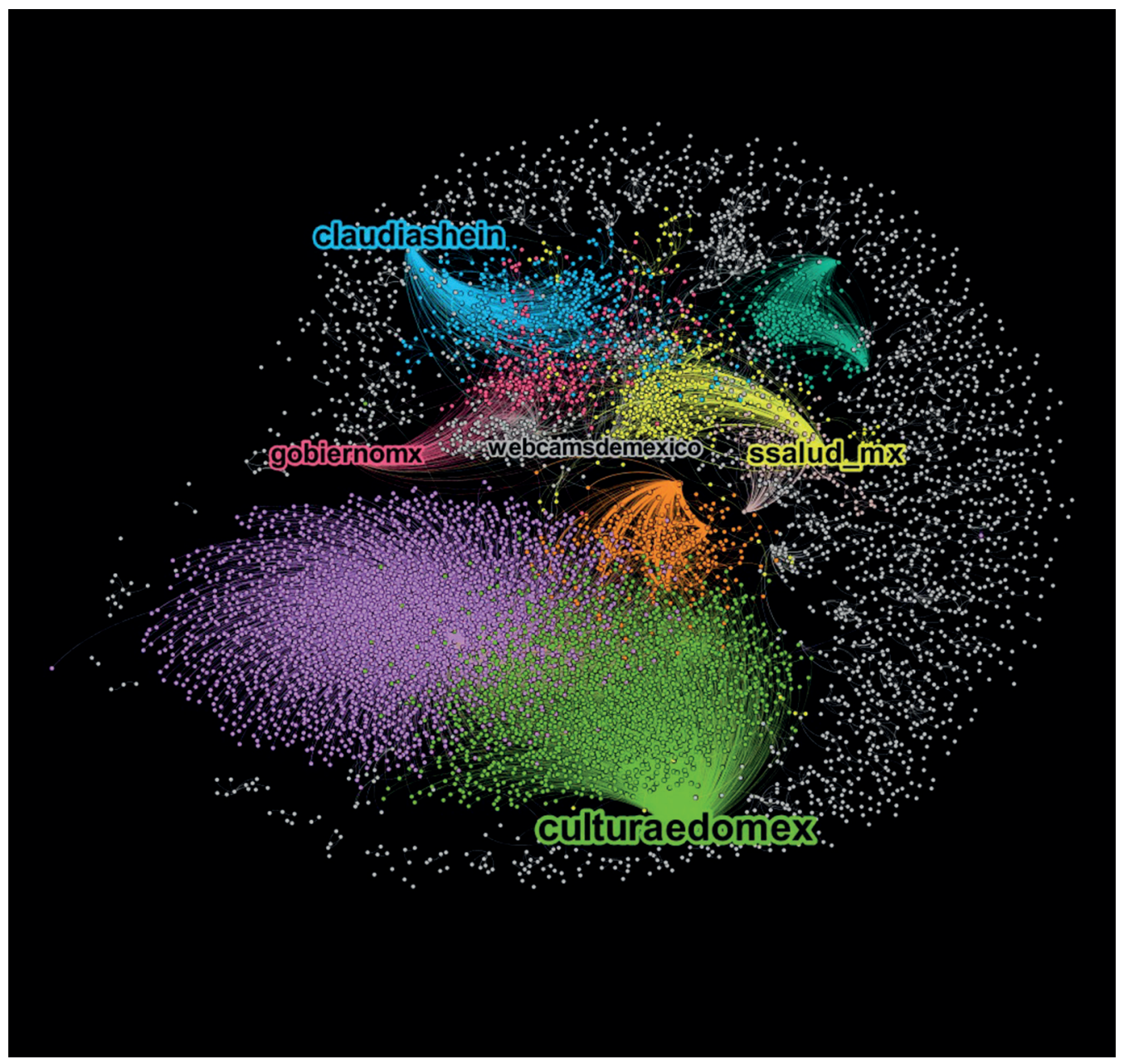

FUENTE: ELABORACIÓN PROPIA CON NODEXL Y GEPHI. 
De esta perspectiva los usuarios cambian, mostrando que la red puede verse e interpretarse de diferentes maneras a partir del nivel de análisis que se desee, debido a que los indicadores son relativamente autónomos entre sí, cuando se estructura la red con base en un indicador, la red puede cambiar de forma y considerar a otros nodos que poseen un atributo específico.

El grado de intermediación permite identificar aquellos nodos que en términos relativos pueden diseminar la información a un público mayor. En la siguiente tabla se observan los indicadores de los cinco nodos con mayor grado de intermediación.

TABLA 3. NODOS CON MAYOR GRADO DE INTERMEDIACIÓN

\begin{tabular}{|l|l|c|c|c|c|}
\hline \multicolumn{1}{|c|}{ NODO } & \multicolumn{1}{|c|}{ ROL } & $\begin{array}{r}\text { GRADO DE } \\
\text { ENTRADA }\end{array}$ & $\begin{array}{c}\text { GRADO DE } \\
\text { SALIDA }\end{array}$ & $\begin{array}{c}\text { NIVEL DE } \\
\text { GRAD0 }\end{array}$ & $\begin{array}{c}\text { GRADO DE } \\
\text { INTERMEDIACIÓN }\end{array}$ \\
\hline Culturaedomex & $\begin{array}{l}\text { Institución } \\
\text { pública }\end{array}$ & 1665 & 4 & 1669 & 4696 \\
\hline Claudiashein & Política & 116 & 7 & 123 & 1352.83 \\
\hline Ssalud_mx & $\begin{array}{l}\text { Institución } \\
\text { pública }\end{array}$ & 159 & 3 & 162 & 1196.66 \\
\hline gobiernomx & $\begin{array}{l}\text { Institución } \\
\text { pública }\end{array}$ & 133 & 2 & 135 & 986 \\
\hline webcamsdemexico & Sitio web & 49 & 16 & 65 & 779 \\
\hline
\end{tabular}

Fuente:ELABORACIÓN PROPIA CON DATOS OBTENIDOS DE GEPHI.

De esta manera se puede observar que solo el caso de la cuenta @culturaedomex se presenta entre los más relevantes de ambos indicadores, lo que muestra que su posición destaca en una alta centralidad en tanto esta característica implica que los nodos "deben ser los más activos en el sentido de que tengan el mayor número de lazos con los otros actores de la red o del grafo" (Wasserman \& Faust, 2013, p. 200 ), por lo tanto, si los vínculos que posee son relevantes, también es necesario que dicho nodo interactúe con los demás para poder posicionarse como un nodo con prestigio informativo y diseminador.

En esta tabla se evidencia también la participación de instituciones públicas y actores políticos dentro de la esfera pública nacional, siendo el caso de la jefa de 
Gobierno de la Ciudad de México, Claudia Sheinbaum, quien ha establecido criterios de interacción e información que la visualizan como un nodo que potencia los mensajes en las redes donde se encuentra.

De acuerdo con este indicador, estos nodos participan dentro de los caminos geodésicos para conectar dos nodos cualesquiera dentro de la red; así, mientras esté en más caminos, su potencialidad de diseminación aumenta. Por ejemplo, la cuenta oficial de la Secretaría de la Cultura y el Deporte del Estado de México participa en 4696 caminos geodésicos; su relevancia en el flujo de información deriva de sus vínculos que le permiten difundir la información a un número mayor de personas en comparación con otros nodos dentro de la red.

\section{DISCUSIón}

A partir de los datos obtenidos en cada red se pueden destacar elementos relevantes que le dan sentido tanto a la red como a la vinculación simbólica que se ha construido alrededor de \#QuédateEnCasa.

La centralidad de un nodo cambia conforme los indicadores de la red. Sea por el término de vínculos o por su función de diseminador, la red expresa los procesos de interacción social mediante la información que allí se encuentra en permanente flujo.

A nivel simbólico, el hashtag \#QuédateEnCasa se constituyó en una forma creativa, clara y específica sobre la mejor y más importante forma de disminuir el riesgo de contagio, esto es, mantenerse en los hogares y evitar salir a menos de ser indispensable; la construcción simbólica del hashtag fue reforzada con la imagen del corazón debajo de un techo que en conjunto delimitan la connotación del mensaje emanado de las distintas dependencias y funcionarios públicos.

Ese producto visual representa el lado emotivo y soft de la medida gubernamental; no es un toque de queda, ni una represión emanada del Estado, sino una solicitud cuyo único beneficio es la salud de la propia población. El carácter imperativo de la oración: "Quédate en casa" se suaviza para convertirse en una oración desiderativa en la que más que una orden/obligación se desea/anhela que el receptor haga algo. Con esto, la vinculación del corazón dentro de una casa intenta fortalecer este cambio de sentido mediante la analogía entre la salud/ bienestar en el hogar, que sirve de refugio. 
FIGURA 6. RECURSO VISUAL DE \#QUÉDATEENCASA

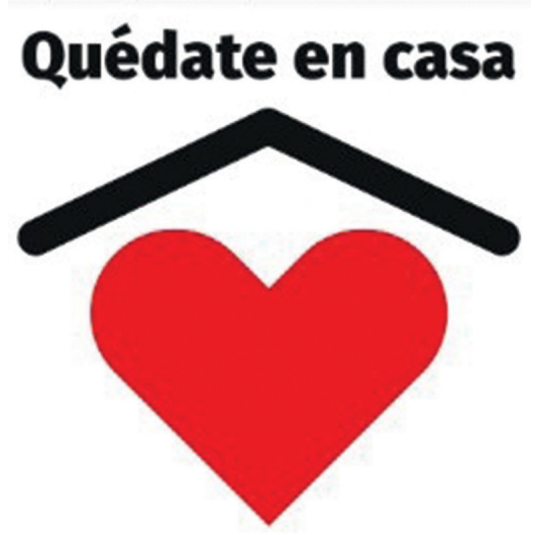

FUENTE: https://coronavirus.gob.mx/quedate-en-casal

Esta relación simbólica ha sido efectiva para evitar reconocer negativamente esta medida, aunque ello no ha impedido que parte de la población salga por cuestiones laborales o de salud, que se propaguen fake news sobre esta y otras medidas tomadas en México, o por el sesgo mediático y político que ha recibido esta estrategia con la finalidad de diezmar los esfuerzos de la actual administración para enfrentar este contexto adverso.

Las notificas falsas adquieren relevancia en la red no solo por el fin desinformativo que presentan, sino por el anonimato que adquieren en la infinitud de su reproducción en la red. De esa manera "es ese gran espacio virtual el que también permite el desvanecimiento de responsabilidades informativas, ya que la premura de publicar información reduce la capacidad y relevancia de verificar las fuentes que sustenten el hecho social mercantilizado en información" (Olmedo, 2020 b, p. 163).

Las notificas falsas o fake news potencian su alcance mediante el conectar con aquello que los usuarios piensan sobre un tema, pero que no encuentran fuentes para sustentarlo; de allí que las noticias falsas son compartidas por aquellos usuarios que ven allí reflejado parte de su pensamiento sobre el hecho social, independientemente que dicha opinión esté sustentada por investigaciones, datos científicos o análisis críticos sobre el tema en cuestión, en este caso la covid-19.

Finalmente, a partir de este análisis se ha podido observar que el hashtag \#QuédateEnCasa ha sido promovido en su mayoría por instituciones públicas y 
actores políticos que poseen una relevancia en la esfera pública, sea por el cargo que ostentan en los estados o por su responsabilidad en el ámbito público para informar a sus respectivas poblaciones. Esta característica ha sido relevante en tanto que son las instancias del Estado quienes promueven la información oficial sobre el avance de la pandemia y las medidas a tomar por la ciudadanía.

\section{CONCLUSIONES}

A lo largo del presente trabajo se ha dado paso al estudio de una estrategia comunicativa que se ha empleado por parte del gobierno mexicano para informar e invitar a la población mexicana a permanecer en sus hogares durante las diferentes etapas de la pandemia de la covid-19.

La construcción del hashtag \#QuédateEnCasa representa un esfuerzo creativo e informativo que sintetiza la necesidad del confinamiento social para reducir el impacto de la pandemia; al ser una estrategia del Estado mexicano materializado en sus diferentes niveles político-administrativos (federal, estatal y municipal), la red evidenció que su promoción y difusión ha sido prácticamente por los diferentes actores políticos e institución públicas que están generando la información oficial sobre los impactos y las medidas tomadas en cada entidad estatal para reducir los daños de la Covid-19.

Si bien las restricciones emanadas de la pandemia han reducido los procesos de socialización en el espacio público, el espacio virtual se ha convertido en un lugar donde los individuos ya no solo interactúan, sino también se informan, por lo que la estrategia convergente empleada por el gobierno federal para informar a la población mediante las redes sociodigitales se ha convertido en una forma de llevar la información oficial de manera directa, con la finalidad de satisfacer la necesidad informacional sobre las medidas tomadas y hacer contrapeso a las fake news que se dispersan de una manera transmedia.

Por otro lado, el uso de este hashtag como variable para la construcción, visualización y análisis de redes adquiere importancia en tanto muestra la estructura que se forma a partir del flujo de información; este método permite que se visualice en términos de interacción-comunicación el impacto de esta estrategia; su uso, por tanto, permite observar las redes que se forman en la red a partir de enlaces que tienen que ver con las dinámicas sociales que se llevan a cabo en dicho espacio. 
El método por sí mismo implica ventajas y desventajas propias del contexto digital en el que se emplea. Entre las ventajas del uso de este método es visualizar los procesos de difusión de la campaña en términos cuantitativos, así como para identificar aquellos aliados que pueden coadyuvar a incrementar el alcance de dicha estrategia comunicativa. En este sentido, su uso permite materializar procesos de comunicación que llevan a cabo entre las cuentas/perfiles que se (re) conocen mediante la interacción con la información.

En términos de desventajas es importante mencionar que en la red pueden estar presentes cuentas falsas o bots que participen de manera positiva/negativa para la estrategia; además de ello, el propio análisis debe considerar que aquellas personas que interactúan mediante Twitter deben poseer un dispositivo electrónico y acceso a internet para poder conocer y ser parte de este tipo de interacciones, lo cual reduce el trabajo de análisis a espacios mayoritariamente urbanos y en transición, dificultando la participación de población en zonas rurales que no cuentan con los elementos necesarios para ser/estar en la red.

Finalmente, este análisis deja claro que con la inserción de las redes sociodigitales en la vida cotidiana, las posibilidades de su análisis se amplían conforme el propio objeto de estudio se complejiza. Las relaciones sociales se replican en el mundo virtual para fortalecerse o incrementarse hasta el límite que se fije el propio individuo.

\section{REFERENCIAS}

Barabási, A.-L. (2011). Introduction and Keynote to A Networked Self. En Papacharissi, Z. A Networked Self (pp. 1-14). Routledge.

Berardi, F. (2020,16 de marzo). Crónica de la psicodeflación. En Amadeo, P. (ed). Sopa de Wuhan (pp. 35-54). ASPO.

Contreras, L. (2017). Las redes sociales digitales como factor de proximidad entre la sociedad y los gobiernos subnacionales en México Digital. RICSH. Revista Iberoamericana de las Ciencias Sociales y Humanísticas, 6(12), 1-26. https://doi.org/10.23913/ricsh. v6i12.131

Criado, I. J., \& Gil-García, R. J. (2013). Gobierno electrónico, gestión y políticas públicas: Estado actual y tendencias futuras en América. Gestión y Política Pública, 13, 3-48.

Cruz, A. (2020, 23 de marzo). Lanza gobierno capitalino la campaña Quédate en casa. La Jornada. https://www.jornada.com.mx/ultimas/capital/2020/03/23/lanza-gobierno-capitalino-la-campana-quedate-en-casa-5577.html 
DaHLGren, P. (2012). Mejorar la participación: la democracia y el cambiante entorno de la web. En Champeau, S. y Innerarity, D. Internet y el futuro de la democracia (pp. 4567). Paidós.

García, C., \& Olmedo, R. A. (2019). El nuevo opio del pueblo: apuntes desde la Economía Política de la Comunicación para (des)entender la esfera digital. Iberoamérica Social, 7(XII), 84-96. https://iberoamericasocial.com/wp-content/uploads/2019/07/0lmedo-R.-2019.-El-nuevo-opio-del-pueblo-apuntes-desde-la-econom\%C3\%ADa-pol\%C3\%ADtica-de-la-comunicaci\%C3\%B3n-para-desentender-la-esfera-digital.pdf

Granovetter, M. S. (1973). The strength of weak ties. American Journal of Sociology, 78(6), 1360-1380. https://doi.org/10.1086/225469

Gutiérrez, J. (2020, 6 de mayo). Mexicano están 42\% más tiempo en redes sociales en cuarentena: Nielsen. La Jornada. https://www.jornada.com.mx/ultimas/economia/ 2020/05/06/mexicanos-estan-42-mas-tiempo-en-redes-sociales-en-cuarentena-nielsen-1173.html

Han, B. (2016). En el enjambre. Herder.

Hanneman, R. (2000). Introducción a los métodos del análisis de redes sociales. Centro de Investigaciones Sociológicas.

Lozares, C. (1996). La teoría de redes sociales. Papers, 103-126.

Luna, A. (2020,3 de mayo). ¿Tú conocer a alguien que se haya muerto de CoVID-19?: por qué hay mexicanos que no creen en la pandemia. Infobae. https://www.infobae.com/ america/mexico/2020/05/03/tu-conoces-a-alguien-que-se-haya-muerto-de-covid-19por-que-hay-mexicanos-que-no-creen-en-la-pandemia/

Olmedo, R. A. (2020a). Implicaciones metodológicas sobre el uso del Análisis de Redes Sociales en redes sociodigitales. Quórum Académico, 17(2), 73-94. http://produccioncientificaluz.org/index.php/quorum/article/view/32758

Olmedo, R. A. (2020b). La comunicación en la posmodernidad: influencia en el sujeto, campo y medio. Iberoamérica Social (XIV), 157-172. https://iberoamericasocial.com/ wp-content/uploads/2020/06/Olmedo-R.-2020.-La-comunicaci\%C3\%B3n-en-la-posmodernidad-influencia-en-el-sujeto-campo-y-medio.pdf

Olmedo, R. A. (2019). \#AmorEsAmor como constructor de redes digitales en e movimiento LGBTTTIQA en México. Virtualis, 10(19), 109-133

Paniagua, J. A. (2012). Curso de análisis de redes sociales. Metodología y estudios de caso. Universidad de Granada.

Pisani, F., \& Piotet, D. (2007). La alquimia de las multitudes. Paidós.

Ramírez, R. (2020, 28 de marzo). Es nuestra última oportunidad, quédate en casa, advierte López-Gatell. El Sol de México. https://www.elsoldemexico.com.mx/mexico/ coronavirus-mexico-ultima-oportunidad-quedate-en-casa-hugo-lopez-gatell-coronavirus-propagacion-muertes-contagios-5030575.html

Ricaurte, P., \& Ramos-Vidal, I. (2015). Investigación en redes sociales digitales: consideraciones metodológicas desde el paradigma estructural. Revista Virtualis, 165-194. 
Rodríguez, C. A. (2015). Articulación y contrapoder. Los protagonistas del activismo digital en México (2009-2014). En Winocur Iparraguirre, R. y Sánchez Martínez, J. A. (comps.) Redes sociodigitales en México (pp. 81-114). México: Conaculta-FCE.

Rogers, R. (2018). Digital methods for across-platform analysis. En Burgess, J., Marwick, A. y Poell, T. (eds.) The SAGE Handbook od Social Media (pp. 91-110). SAGE Publications.

Rovira, G. (2017). Activismo en red y multitudes conectadas. Icaria.

Torres, L. C. (2013). Cómo analizar redes sociales en internet. El caso twitter en México. En Islas, O. y Ricaurte, P. Investigar las redes sociales. Comunicación total en la sociedad de la ubicuidad (pp.158-169). Razón y Palabra.

Van Dijck, J. (2016). La cultura de la conectividad: Una historia crítica de las redes sociales. Siglo XXI Editores.

Wasserman, S., \& Faust, K. (2013). Análisis de redes sociales. Métodos y aplicaciones. Centro de Investigaciones Sociológicas. 